\title{
GREEN SYNTHESIS AND THE STABILIZATION OF SELENIUM NANOPARTICLES USING CARBOXYMETHYL STARCH
}

\author{
Vishakha VISHAKHA, A. M. ABDEL-MOHSEN, J. JANCAR \\ Central European Institute of Technology, Brno University of Technology, Brno, Czech Republic, EU, \\ vishakha.vishakha@ceitec.vutbr.cz
}

https://doi.org/10.37904/nanocon.2020.3754

\begin{abstract}
The growing interest in biodegradable products paves the way for the safest sustainable earth. Starch is an extensively studied, cost-effective, easily accessible, and highly trusted resource to produce biodegradable products in the present and future. However, the exploitation of these starch in several fields requires substantial changes in its chemical functionalities and related properties. Here, we investigated the conditions $(\mathrm{pH}$, temperature, the concentration of starch, concentration of chloroacetate, time, the ratio between starch/sodium hydroxide, etc.) that affect the preparation of carboxymethyl starch (CMS). The chemical structure and degree of substitution of native starch and CMS were confirmed by Fourier transform infrared ( FTIR), X-ray powder diffraction (XRD), Thermogravimetric analysis (TGA), potentiometric titration, Scanning electron microscope (SEM), and Nuclear magnetic resonance spectroscopy (NMR ). CMS's rheological properties show that CMS's intrinsic viscosity increased with increased degrees of substitutions (DS) from CMS $(0.05$ to 0.45$)$. The CMS with different DS $(0.05$ to 0.45$)$ was used for the first time to stabilize selenium nanoparticles (Se-NPs), showing spherical shape with a high homogenous size of Se-NPs (approx. $50 \mathrm{~nm}$ ). The NPs shape and size stability were investigated and confirmed by different techniques like Dynamic light scanning (DLS), SEM, and Transmission electron microscope (TEM).
\end{abstract}

Keywords: Selenium nanoparticles, carboxymethyl starch, starch

\section{INTRODUCTION}

Sodium carboxymethyl starch was first prepared in 1924. Since then, many derivatives have been prepared using the starch's different sources such that potato, amaranth, rice, mungbean, etc. These starches have variations in their amylose content, which is the primary cause of the different substitution degrees. Chemical modification of starch in this way could be useful for the textile, pharmaceutical industries, food industries, absorbents, and adhesives, etc. Nowadays, researchers try to make the material from the CMS for the cell carrier using inorganic material insertion. The carboxymethyl starch (CMS) is prepared by starch's reaction with the sodium monochloroacetate (SMCA) or monochloroacetate (MCA) in the presence of $\mathrm{NaOH}$. Generally, this reaction occurs using a heterogeneous medium such as (ethanol/water, isopropyl alcohol/ water, benzene/ water), etc. This is because the response between starch and SMCA follows the Williamson ether synthesis and is based on the $\mathrm{SN}^{2}$ mechanism. The polar aprotic solvent is required to do etherification efficiently. The substitution factor is solvent, the concentration of the $\mathrm{NaOH}, \mathrm{SMCA}$, temperature, and time $[2,3]$.

The solvent medium is mostly partially water because Carboxymethyl starch's solubility in water starts from 0.1 DS. Many researchers have claimed that using isopropanol as the organic solvent with water is the most prominent solvent for the higher degree of substitution (DS) in CMS. They have used many combinations of the organic solvent and water such as methanol/water, ethanol/water, isopropyl alcohol/water for the reaction. They have claimed that isopropyl alcohol/water mixture is one of the best heterogeneous solvents for the carboxymethyl starch with an intact granule size. It is easy to remove unreactive and impurities from CMS [1]. 
The temperature is also the primary cause of changing the starch's properties because gelatinization occurs in starch at higher temperatures. Still, the conditions may vary according to the percentage of the amylose content in the source. Potato starch is an abundant source with $28 \%$ amylose content, and its gelatinized at $\sim 65^{\circ}-80^{\circ} \mathrm{C}$ in the water. Choosing a suitable solvent also plays a crucial role in the reaction because it needs to dissolve the reactants.

The solvent's concentration affects the solubility of the $\mathrm{NaOH}$ during the reaction because it is completely soluble in water but does not soluble in isopropyl alcohol at room temperature. The alkylation of the starch needs to be dissolved in the solvent. Otherwise, the separation of the two layers becomes broader in the reaction mixture. Moreover, penetration of the $\mathrm{NaOH}$ in the starch is impossible, leading only to surface annealing. Therefore $10 \%$ of isopropyl alcohol is recommended for the highest DS. Concerning the amylose content present in the starch, the $\mathrm{NaOH}$ concentration has also demonstrated the primary factor for increasing the DS because the complete substitution of the SMCA is based on the catalyst $(\mathrm{NaOH})$. As many researchers reported, changing the ratio of the SMCA: $\mathrm{NaOH}$ shows a significant effect on the DS. There is more chance to do side reaction rather than the primary reaction during the CMS's preparation. The side reaction may compete with the immediate response and leads to a decrease of DS.

Researchers have used the synthesis of Se-NPs by ascorbic acid at room temperature in the past few years, as it is a convenient and eco-friendly method $[6,8]$ and for the stabilization of the nanoparticles used polysaccharides such as cellulose and its derivatives [7]. This study's main aim was to stabilize the Se-NPs by using synthesized CMS as polysaccharides act as stabilizers, facilitated by inter-and intra-hydrogen bonding and resulting in a template that binds the nanoparticles and avoids them to aggregate [4,5]. Moreover. In the synthesis of selenium nanoparticles (Se-NPs), the capping agent has a crucial role in influencing the nanoparticle characteristics. Starches are abundant natural biopolymers, inexpensive, and renewable. Starches are composed of polymers that contain amylose and amylopectin. Amylose and amylopectin have the functional molecules before and after modification, acting as a selenium nanoparticles template [4].

\section{EXPERIMENTAL SECTION}

\subsection{Material and Reagent}

All chemicals were purchased from the Sigma Aldrich, Penta chemicals and used as received. Potato starch is purchased from the Czech Republic. The Bruker FT-IR was used for the Fourier transformation infrared in attenuated total internal reflectance $400 \mathrm{~cm}^{-1}$ to $4000 \mathrm{~cm}^{-1}$ at room temperature. Thermogravimetry analysis was carried out on the Trios instrument using $\mathrm{N}_{2}$ as carrier gas with a temperature ramp is 10 degrees /min from room temperature to 700 degrees.

\subsection{Carboxymethylation of the Potato Starch}

$1 \mathrm{~g}$ of starch ( $6 \mathrm{mmol}$ ) was added to the $250 \mathrm{ml}$ dried round bottom flask and mixed with $45 \mathrm{ml}$ isopropyl alcohol and $5 \mathrm{ml}$ distilled water (9:1). Followed by the dropwise addition of the $10 \% \mathrm{NaOH}(2.5 \mathrm{M})$ solution $(\mathrm{w} / \mathrm{v})$ for $60 \mathrm{~min}$ by noticing the $\mathrm{pH}(8-12)$ with stirring at $(500 \mathrm{rpm}-900 \mathrm{rpm})$ at room temperature, continuing the stirring for $6 \mathrm{~h}$. After $6 \mathrm{~h}$, the addition of an aqueous solution of SMCA ( $6 \mathrm{mmol}$ to $18 \mathrm{mmol})(\mathrm{w} / \mathrm{v})$ for $120 \mathrm{~min}$ at 50 degrees Celcius. Once the addition of the SMCA was done, then the reaction was continued for 1 hour at the above temperature. Followed by the Neutralization with $6 \mathrm{M} \mathrm{HCl}(5.5$ to $6 \mathrm{pH})$, and the filtration was done by cold $90 \%$ ethanol $/ 85 \%$ ethanol for several times filtration to remove the chlorides. The degree of substitution was calculated by the back titration followed by the complexometric titration. The morphology of the CMS and their shape after a modification has been confirmed by SEM analysis, and the XRD analysis was used to confirm the loss of crystallinity after carboxymethyl group substitution on the potato starch. 


\subsection{Preparation of the Selenium Nanoparticles}

The addition $(5-12 \mathrm{ml})$ of $12.5 \mathrm{mM}$ ascorbic acid into the $25 \mathrm{ml}$ round bottom flask containing $(1-2 \mathrm{ml}) 25 \mathrm{mM}$ sodium selenite solution, which was stirred at room temperature for an hour, until the color changed colorless to orange radish. The appearance of color was due to the reduction of selenium ions into the atoms and for the stability of the nanoparticles, added $0.2 \%$ of CMS. It was stirred for 5 hours until the solution got homogenized. Figure 1 is to illustrate the presence of Se-NPs. The SEM images confirmed the size and shape of the Se-NPs.

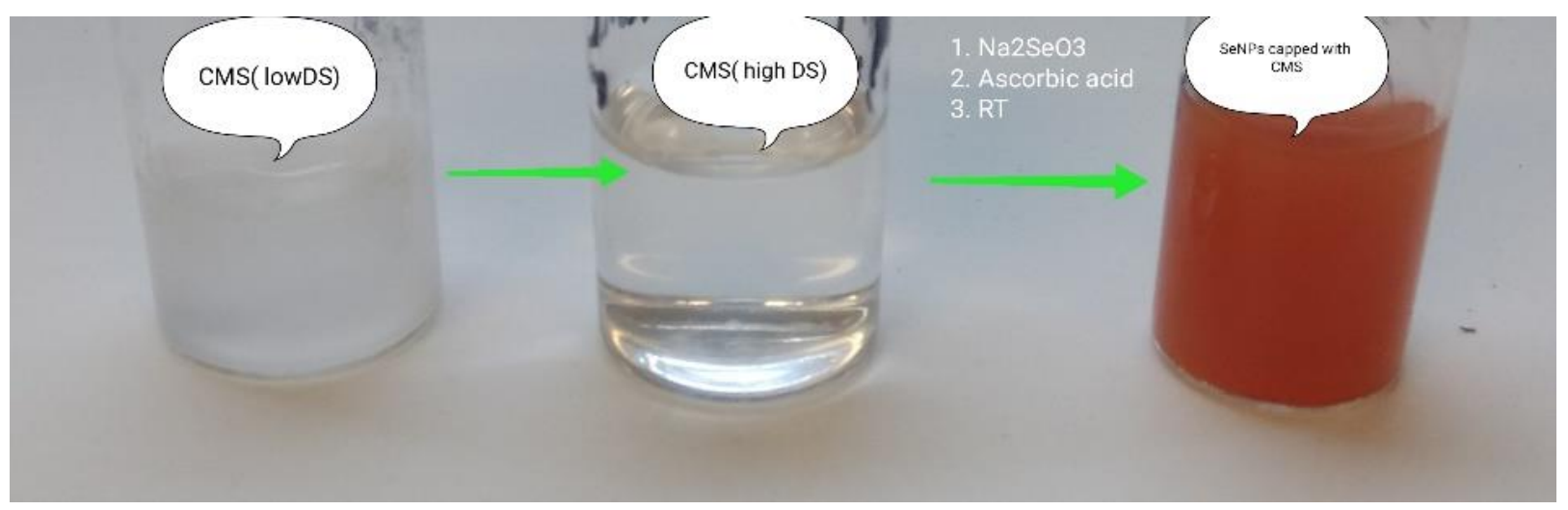

Figure 1 Visibility comparison of CMS with high, low DS, and with Se-NP

\subsection{Back titration}

CMS samples should be acidic and taken $0.02 \mathrm{~g}$ of sample for each measurement and dissolved in $6 \mathrm{ml}$ of 0.1 $\mathrm{M} \mathrm{NaOH}$ and titrated the solution with $0.1 \mathrm{M} \mathrm{HCl}$. The average of the readings has been included for the calculation of the DS.

$W_{C H 2 C O O}=\frac{\left(V_{b}-V_{s}\right) M_{H C l} m_{C H 2 C O O}}{m_{s}} \times 100$

$D S=\frac{162 \times W_{\text {CH } 2 \mathrm{COO}}}{\left(100-W_{\text {CH } 2 \text { COO }}\right) m_{\text {CH } 2 \mathrm{COO}}}$

Where:

$V_{b}$ - blank volume,

$V_{s}$. - sample volume

$m_{s}$ - Mass of the sample in milligram

\subsection{Complexometric titration}

In this titration, $0.02 \mathrm{~g}$ of each sample of CMS were taken in the vials and dissolved in the minimum amount of water, and after the dissolution, precipitation occurs by the addition of the excess of $\mathrm{CuSO}_{4} .5 \mathrm{H}_{2} \mathrm{O}$ solution and titrated against the EDTA (Ethylene diamine tetraacetate)

$n_{\text {CH2COONa }}=\frac{\left(V_{b}-V_{S}\right) \times 2 \times C}{1000}$

$D S=\frac{162 \times n_{\mathrm{CH} 2 \mathrm{COON} a}}{\mathrm{~W}-80 \times n_{\mathrm{CH} 2 \mathrm{COON} a}}$

2- Mole ratio of the $\mathrm{CH}_{2} \mathrm{COONa}$ / Initial moles of the EDTA, $\mathrm{C}$ is the EDTA concentration $(0.01 \mathrm{M})$, and $\mathrm{W}$ signifies the weight of the sample in gram. 


\section{RESULTS}

\subsection{Overview of the result}

Percentage yield, reaction efficiency, and substitution were analyzed by the experiment performed under the constant temperature and with the definite $\mathrm{pH}$. The reaction's efficiency decreased as we exceed the ratio of SMCA over the 1:1 molar ratio because of the concentration of sodium glycolate and the unreactive SMCA increases. The molar ratios: $1: 0.5,1: 1,1: 1.5,1: 2,1: 3$ and their percentage yields are $57.55 \%, 49.8 \%, 42.01 \%$, $37.3 \%$, and $30 \%$ respectively and their DS is $0.10,0.13,0.47,0.50$ and 0.62 by back titration. These all ratios are soluble in water at room temperature except the 1:0.5 molar ratio.

\subsection{Analysis of the carboxymethyl starch}

FT-IR analysis of CMS is illustrated in Figure 2. The sharp band comes around $1600 \mathrm{~cm}^{-1}$, and the 1400 $\mathrm{cm}^{-1}$ shows a carboxylic group where an unsymmetric stretching peak comes at $1600 \mathrm{~cm}^{-1}$, and the symmetric stretching peak is visible at about $1400 \mathrm{~cm}^{-}$ 1. (COO-) Moreover, the unmodified portion was shown under the range of the $1200 \mathrm{~cm}^{-1}$ to $500 \mathrm{~cm}^{-1}$. Figure 3 is presenting the effect of $\mathrm{pH}$ on the DS. When the $\mathrm{pH}$ increases up to some level, DS also increases. Figure 4 illustrates the effect of the concentration of (starch: SMCA) on the \%yield of the CMS, and Figure 5 defines the impact of SMCA on the carboxymethyl percentage on the starch.

Figure 2 FT-IR spectra demonstrating the presence of carbonyl peaks
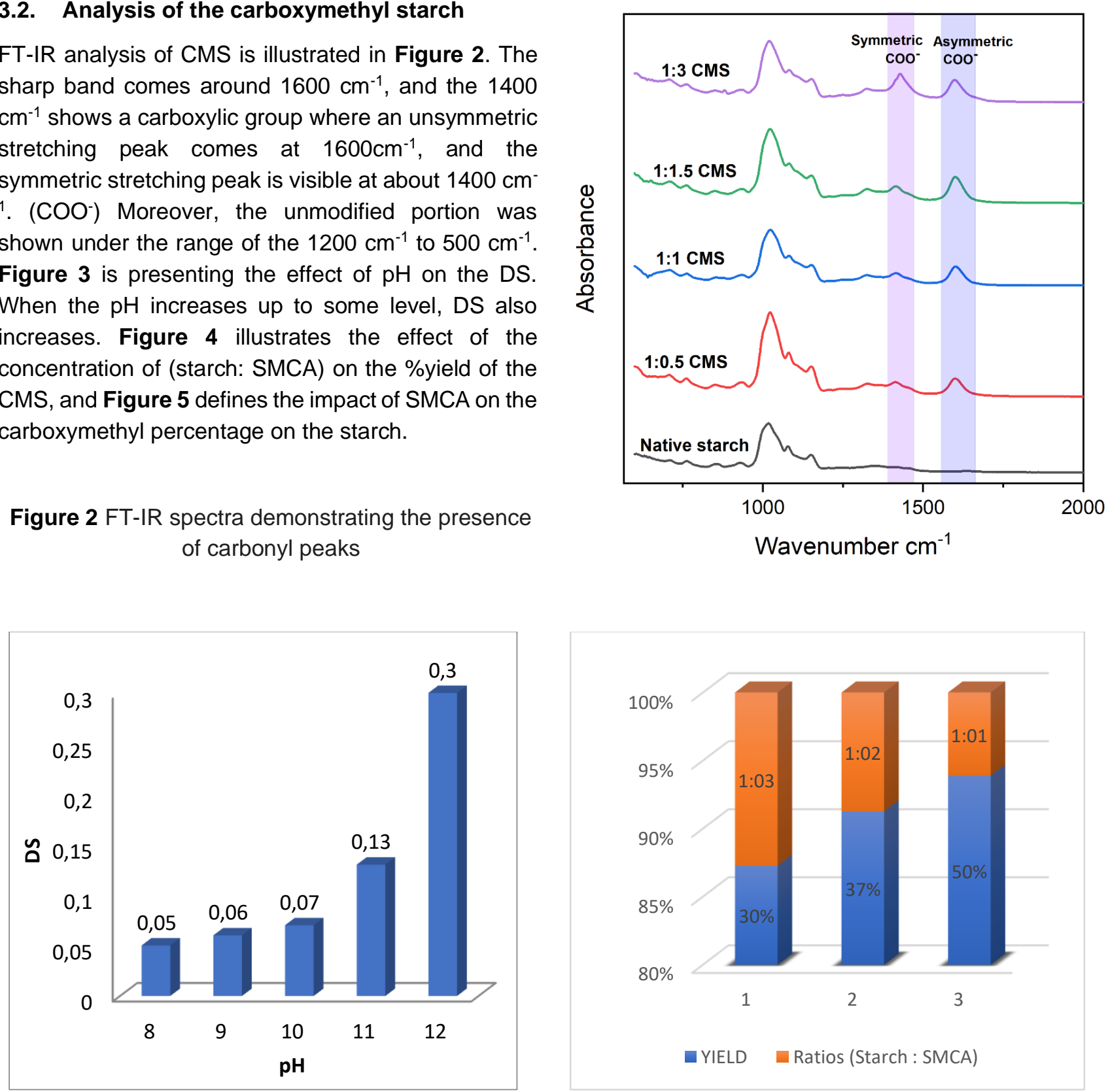

Figure 3 Bar graph is demonstrating the effect of $\mathrm{pH}$ on the degree of substitution

Figure 4 Bar graph is demonstrating the effect of the starch: SMCA ratio on the \%yield of CMS 


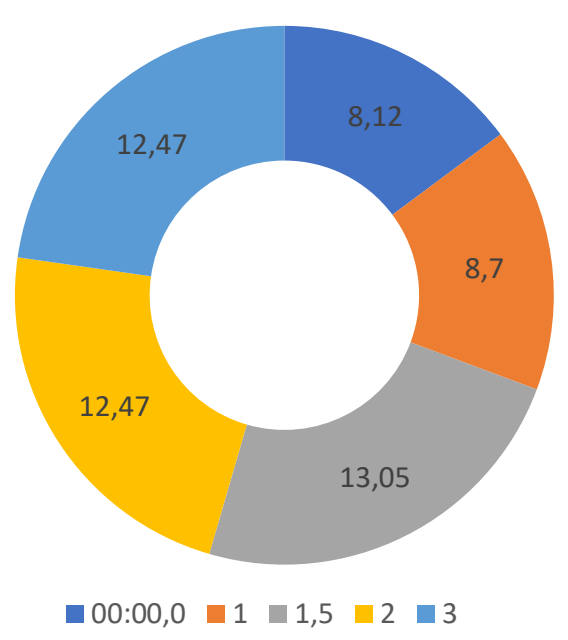

Figure 5 Pie-chart is demonstrating the effect of the amount of SMCA on the carboxymethyl substitution

\subsection{X-Ray Diffraction Analysis}

X-ray powder diffractometer rigaku smart lab $3 \mathrm{kw}$ was used with automatic $\mathrm{X}$-ray powder diffractometer with $\Theta / \Theta$ goniometer. The brag-Brentano and parallel beam modes are complemented by the use of additional asseries. Diffractometer measurement has been taken at 40 kilovolts, current 30 milliamperes. In Figure 6, all samples' showed no sharp peak, which illustrates the loss of crystallinity after the chemical modification, which indicates that substituted carboxymethyl groups deform the crystallinity during the chemical modification. The small sharp peak that appeared in one of the ratios should be of impurity. The degree of crystallinity decreases as the substitution degree increases. Figure 7 depicts crystalline peaks at 28.6 and 42.9 degrees, which confirm the presence of Se-NPs embedded with CMS; in this figure, the XRD pattern shows some characteristics peaks of Se-NPs that confirm the presence of $\mathrm{Se}(0)$. The reason for having low-intensity peaks and less due to the CMS's high viscosity might hide the minimal peaks.

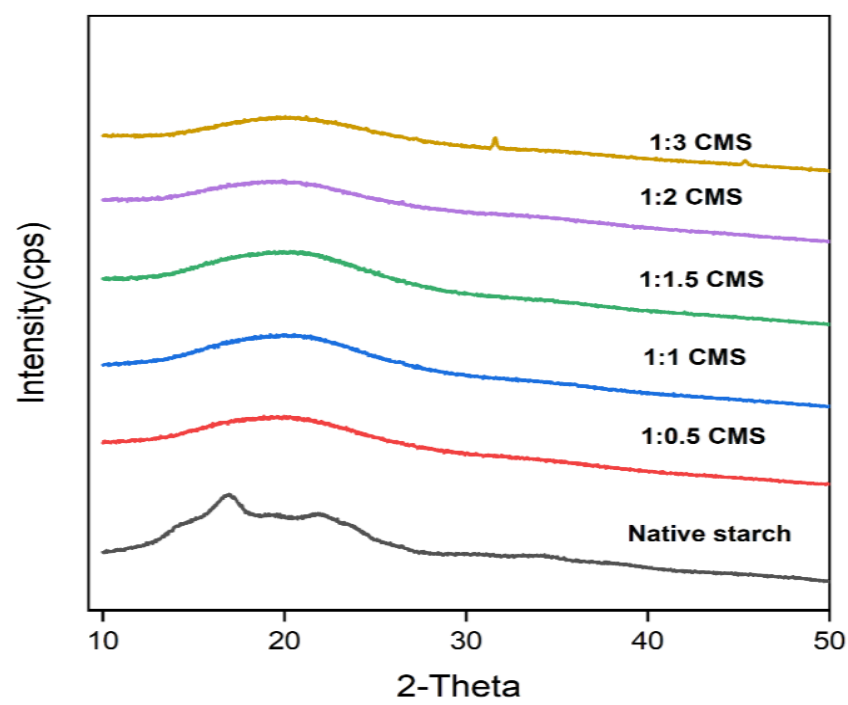

Figure 6 XRD spectra demonstrating the loss of crystallinity of the CMS ratios with respect to the native starch

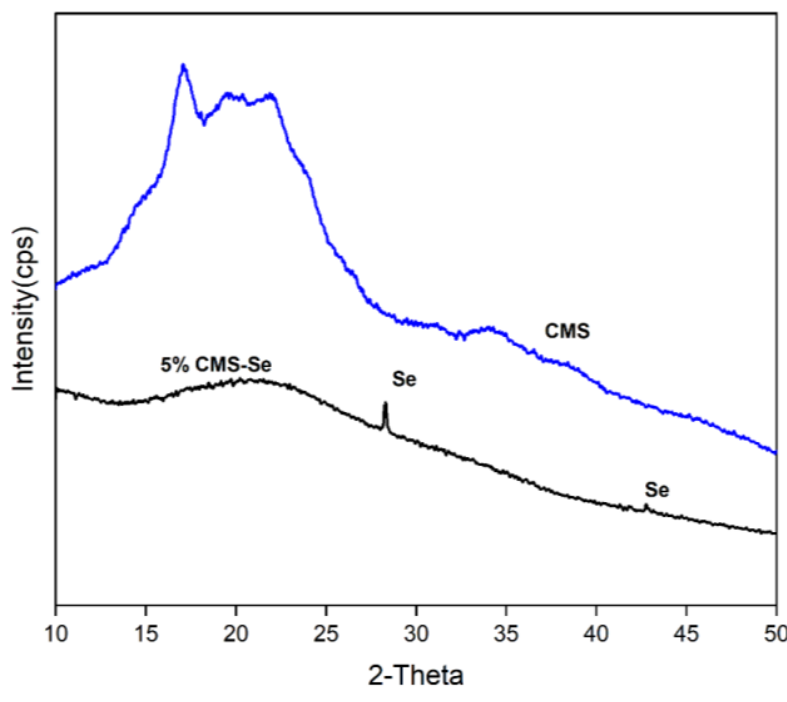

Figure $7 \mathrm{XRD}$ spectra demonstrating the characteristic peaks of Se-NPs embedded in CMS 


\subsection{Investigating the Surface Morphology (SEM)}

Figure 8 illustrates the surface morphology of native starch, CMS, and Se-NPs capped with CMS.which was studied using a scanning electron Microscope. Figure (a) shows the shape of the potato starch granules, spherical, and almost all regular in shape. Figure (b) shows the change in the shape of the potato starch granule after modification by carboxymethylation, size has increased, and shape has distorted. Figure (c) and (d) show the spherical shape of the Se-NPs present on CMS's surface. We have used, The Thermo Fisher (VARIOS) 460 L Field -emission scanning electron microscope (FESEM), which offers sub-nanometer resolution over a wide energy range $(0.7 \mathrm{~nm} @ 1 \mathrm{keV}, 0.6 \mathrm{~nm} @ 2-30 \mathrm{keV})$ with excellent materials contrast. The microscope was equipped with a wide array of imaging and analytical detectors for structural and compositional analysis.

a)

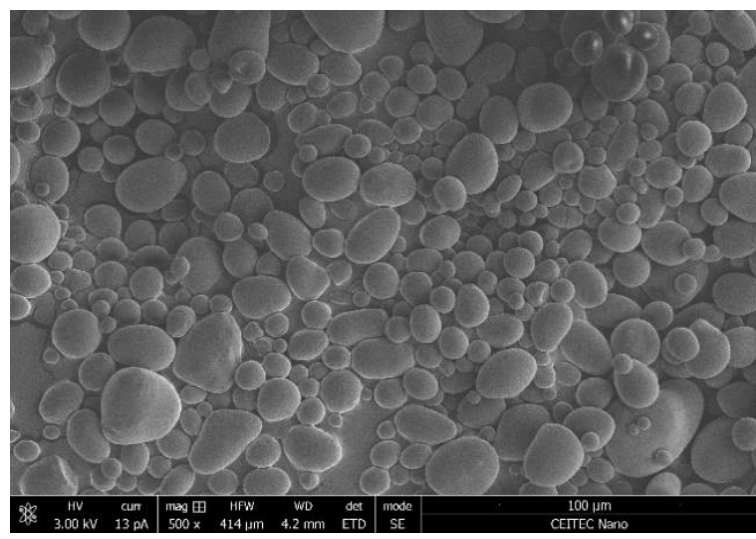

C)

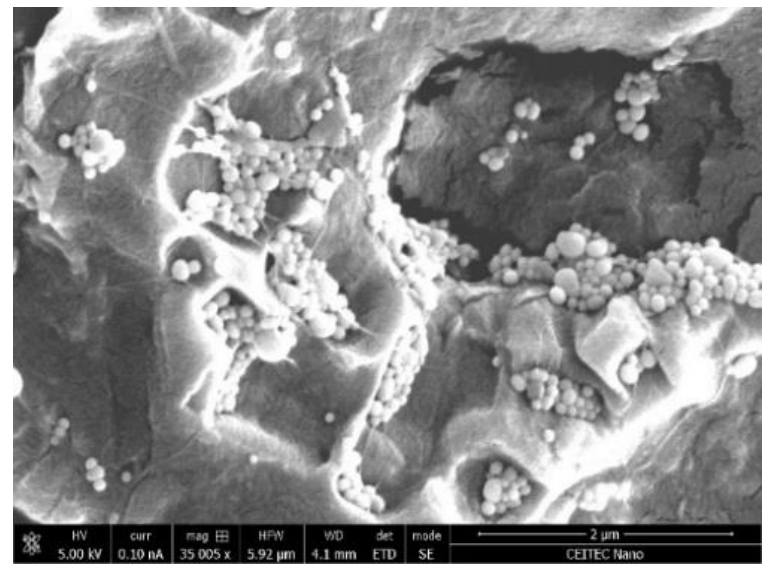

b)

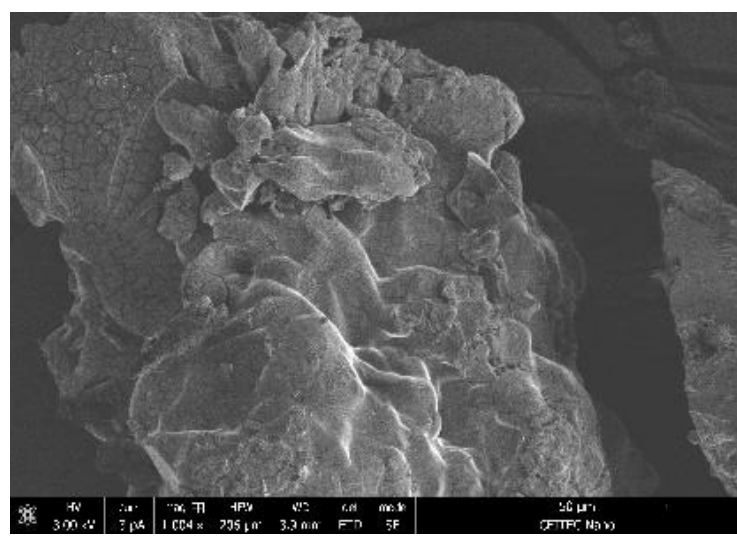

d)

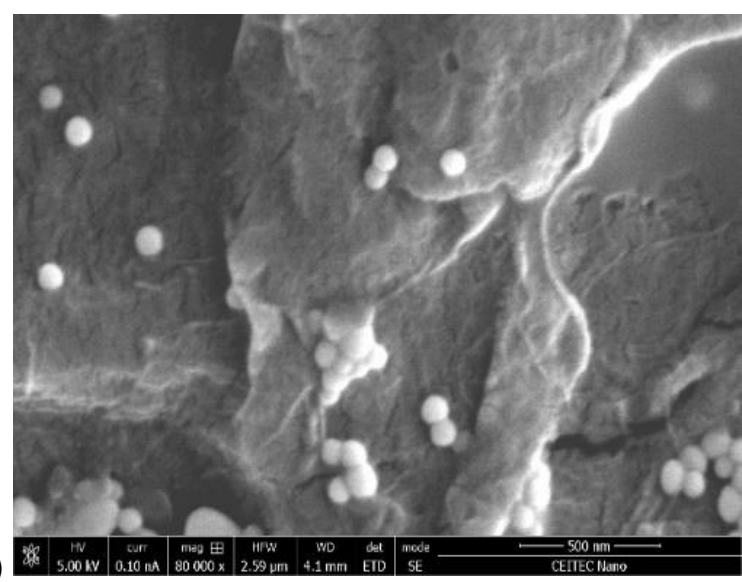

Figure 8 SEM image is demonstrating the shape of Potato starch granules; (a). SEM image is demonstrating the shape of Carboxy;(b). SEM images demonstrate the presence of Se-NPs synthesized by ascorbic acid and capped with CMS;(c) and (d).

\section{DISCUSSION}

In the past, nanoparticles were stabilized by different stabilizers; some are synthetic, and some are biocompatible [7,8]. In this study, we have used the CMS as a capping agent for the Se-NPs, which has a higher capability to stabilize the Se-NPs at room temperature due to the carboxymethyl group, which introduces the chargeable group. Additionally, the charge group's presence makes it best to bind with Se-NPs and hold them firmly for a long without destroying the Se-NPs original size and shape. The SEM technique confirmed the size and shape. 


\section{CONCLUSION}

Various degree of carboxymethyl moiety substitution was successfully carried out using the less harmful solvent, which acted as a heterogeneous medium for the reaction. Our study confirmed that the amount of SMCA played an essential role in CMS's efficiency and yield. We have confirmed the substitution of the $\mathrm{OH}$ group by $\mathrm{CH} 2 \mathrm{COONa}$ in the potato starch by titration, FTIR, and XRD. The best $\mathrm{pH}$ for each substitution of CMS has been found and would be used in our subsequent studies. The low substituted CMS and higher DS were used as a capping agent for the Se-NPs and analyzed the stability of Se-NPs and confirmation of the size and shape by SEM and XRD.

\section{ACKNOWLEDGEMENTS}

This research was carried out under the project CEITEC 2020 (LQ1601) with financial support from the Ministry of Education, Youth, and Sports of the Czechia under the National Sustainability Programme II.

\section{REFERENCES}

[1] HEINZE, T., LIEBERT, T., HEINZE, U., SCHWIKAL, K. Starch derivatives of a high degree of functionalization 9: carboxymethyl starches. Cellulose. 2004, vol. 11, no. 2, pp. 239-245.

[2] HEINZE, T., KOSCHELLA, A. Carboxymethyl ethers of cellulose and starch-a review. Macromolecular Symposia. 2005, vol. 223, no. 1, pp. 13-40.

[3] MASINA, N., CHOONARA, Y. E., KUMAR, P., DU TOIT, L. C., GOVENDER, M., INDERMUN, S., PILLAY, V. A review of the chemical modification techniques of starch. Carbohydrate polymers. 2017, vol. 157, pp. 1226-1236.

[4] WONGMANEE, Kittiyaporn, SUREEPORN, Khuanamkam and SANOE, Chairam. "Gold nanoparticles stabilized by starch polymer and their use as catalyst in homocoupling of phenylboronic acid." Journal of King Saud University-Science. 2017, pp. 547-552.

[5] CHANG, Peter R., et al. "Polysaccharides as stabilizers for the synthesis of magnetic nanoparticles." Carbohydrate polymer. 2011, vol. 83, no.2, pp. 640-644.

[6] VAHDATI, Mahsa and TAHEREH, Tohidi Moghadam. "Synthesis and characterization of Selenium nanoparticlesLysozyme nanohybrid System with Synergistic Antibacterial properties." Scientific reports. 2020, vol. 10, no. 1, pp. $1-10$.

[7] SHIN, Yongsoon, et al. "Synthesis and stabilization of selenium nanoparticles on cellulose nanocrystal." Materials Letters. 2007, vol. 61, no. 21, pp. 4297-4300.

[8] MALHOTRA, Sonam, NEETU, Jha, and KRUTIKA, Desai. "A superficial synthesis of selenium nanospheres using wet chemical approach." Int J Nanotechnol Appl. 2014, vol. 3, pp. 7-14. 